\title{
Scrotal Lipoblastoma with Radiological and Histological Correlation
}

\author{
Lee K. Rousslang ${ }^{1}$ Cole R. Burr ${ }^{1} \quad$ Jonathan R. Wood ${ }^{1}$ \\ ${ }^{1}$ Department of Radiology, Tripler Army Medical Center, Hawaii, \\ United States \\ Address for correspondence Lee Rousslang, MD, Department of \\ Radiology, Tripler Army Medical Center, HI, United States \\ (e-mail: lee.k.rousslang.civ@mail.mil; Irousslang@gmail.com).
}

Indian J Radiol Imaging 2021;31:710-713.

\author{
Abstract \\ Keywords \\ - adipocytic tumor \\ - lipoblastoma \\ - mesenchymal tumors \\ - paratesticular mass \\ - rhabdomyosarcoma
}

Lipoblastomas are rare benign mesenchymal tumors comprised primarily mature adipocytes, which are most commonly found in infants and children younger than 3 years. They are usually found in the extremities, trunk, head, neck, and retroperitoneum, although cases occurring in the scrotum have been reported. Due to its rarity, there is a relative paucity of literature describing its imaging and management. We present a rare case of a scrotal lipoblastoma, and discuss the current imaging strategies to differentiate this adipocytic tumor from other more common paratesticular masses, including aggressive neoplasms such as rhabdomyosarcomas. Knowledge of the radiological appearance of lipoblastoma can provide the correct diagnosis and prevent unnecessary orchiectomy.

\section{Introduction}

Lipoblastomas are rare benign pediatric mesenchymal tumors, and are the second most common pediatric adipocytic tumor after lipomas. ${ }^{1}$ Ninety percent will present before the age of 3 years, with a median age at presentation of 12 months. ${ }^{2}$ Lipoblastomas occurring in the scrotum are very rare, with only 18 cases being reported in the literature to date. ${ }^{1,3-5}$ Treatment in all previous cases of intrascrotal lipoblastoma has been tumor excision with or without orchiectomy. ${ }^{1,3-5}$ If the diagnosis is certain before surgery, a less invasive scrotal approach can be used, as reported in two cases previously, rather than an inguinal approach. ${ }^{3,4}$ There have been no reported cases of metastasis.

The differential diagnosis of intrascrotal lipoblastoma in an infant also includes rhabdomyosarcoma, which is the most common pediatric paratesticular mass. ${ }^{6}$ Unlike lipoblastoma, rhabdomyosarcoma is a highly aggressive malignancy, with more than $40 \%$ having metastasized by the time of presentation. ${ }^{6,7}$ Intrascrotal rhabdomyosarcomas require radical orchiectomy, and in patients older than 10 years, retroperitoneal lymph node dissection. ${ }^{8}$

Because of its rarity, there is little awareness of intrascrotal lipoblastoma, its imaging characteristics, and its appropriate management. Previous prospective studies demonstrated a lack of consistent imaging protocols of the lesion. ${ }^{9}$ Moreover, lipoblastomas can be difficult to diagnose preoperatively due to similarities in location, clinical presentation, and patient demographics with rhabdomyosarcoma. As a result, all but two previous cases were excised via an inguinal approach. ${ }^{1,3-5}$

Knowledge of current testicular imaging paradigms, including ultrasound (US) and magnetic resonance imaging (MRI), as well as newer, advanced US techniques such as contrast-enhanced ultrasonography (CEUS), and sonoelastography, can assist the radiologist in making the diagnosis, and ensuring the proper surgical approach of the lesion. ${ }^{10}$
DOI https://doi.org/ $10.1055 / \mathrm{s}-0041-1736395$ ISSN 0971-3026. (c) 2021. Indian Radiological Association. All rights reserved.

This is an open access article published by Thieme under the terms of the Creative Commons Attribution-NonDerivative-NonCommercial-License, permitting copying and reproduction so long as the original work is given appropriate credit. Contents may not be used for commercial purposes, or adapted, remixed, transformed or built upon. (https://creativecommons.org/ licenses/by-nc-nd/4.0/)

Thieme Medical and Scientific Publishers Pvt. Ltd., A-12, 2nd Floor, Sector 2, Noida-201301 UP, India 


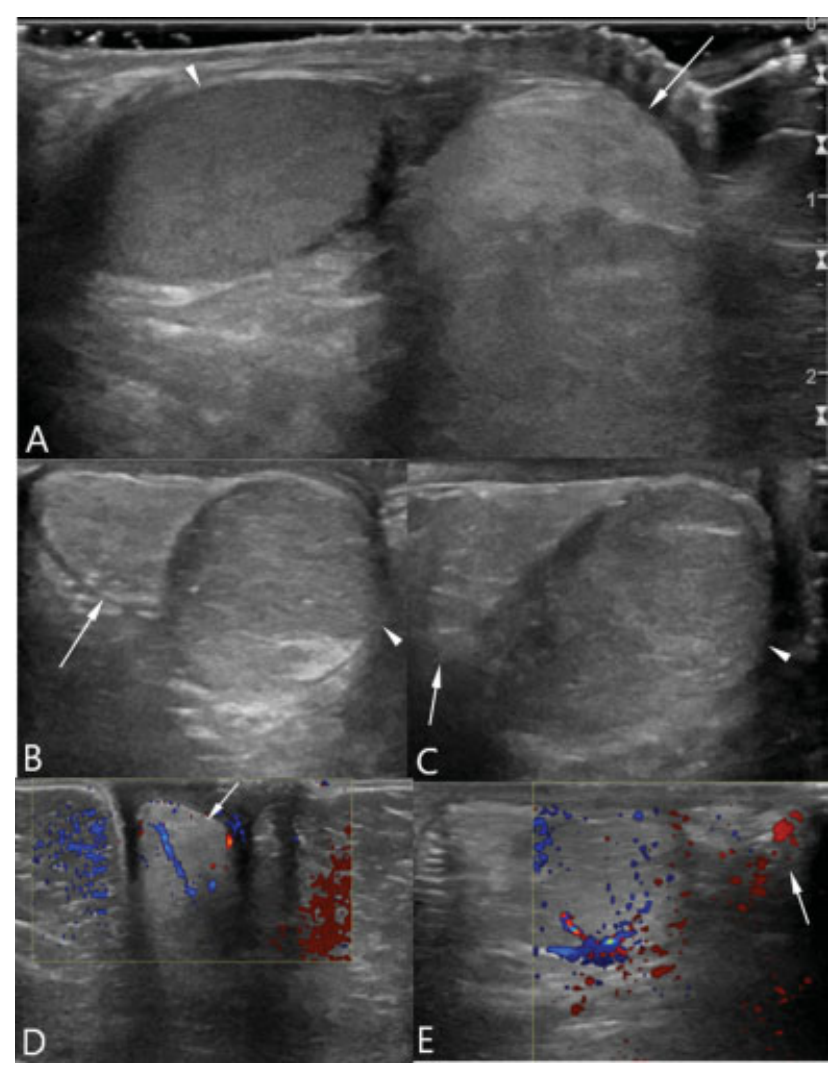

Fig. 1 (A-E) Right testicular ultrasound in transverse view (A) demonstrates a hyperechoic $2.9 \times 1.7 \times 1.7 \mathrm{~cm}$ mass (arrow) seen inferior to the right testicle (arrowhead). Transverse (B) and longitudinal $(C)$ views of the same lesion demonstrate a circumscribed, lobulated, heterogenous mass, shown on pathology to be a lipoblastoma. Various transverse angles (D) and (E) demonstrate intratumoral vascularity (arrows).

\section{Case Presentation}

A 14-month-old previously healthy boy presented with a growing right scrotal mass, present for 1 month with no associated symptoms. The mass was firm, nontender, and separate from the right testicle. Laboratory values, including $\alpha-1$-fetoprotein and beta human chorionic gonadotropin tumor markers, were within normal limits. The patient was referred to pediatric oncology and urology. Scrotal US demonstrated a heterogeneous, hyperechoic, solid, lobulated, oval, well-circumscribed paratesticular mass measuring $2.9 \times 1.7 \times 1.7 \mathrm{~cm}$ (-Fig. 1). Ultrasonography with color Doppler demonstrated the lesion to be hypovascular relative to the adjacent testicle, but hypervascular compared with subcutaneous fat, or a typical lipoma. Imaging modalities including MRI, CEUS, and sonoelastography were not performed. Despite the indeterminate imaging findings, concern for rhabdomyosarcoma remained high, and a right radical orchiectomy with tumor excision was performed. Pathologic evaluation revealed a lipoblastoma (-Fig. 2).

\section{Discussion}

Intrascrotal lipoblastomas are extremely rare, with fewer than 20 ever reported. ${ }^{1,3-5}$ They usually present as a painless,

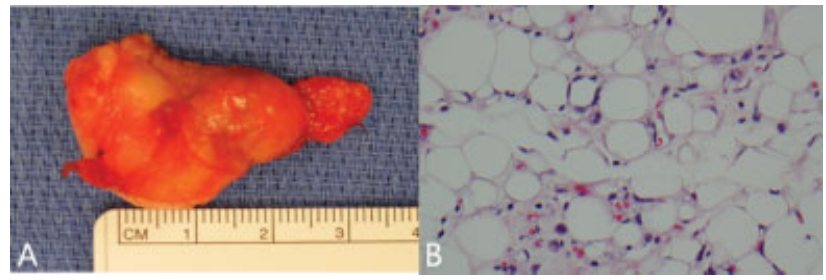

Fig. 2 (A, B) Gross pathology specimen (A) demonstrates a lobulated, yellow, firm mass. Microscopic analysis (B) demonstrates lipoblasts with little to no nuclear atypia, interspersed between normal appearing lipocytes.

growing scrotal mass within the first year of life. ${ }^{1,2}$ Although benign, they can demonstrate locally invasive features or mass effect on adjacent structures. ${ }^{2,9}$ Up to $61 \%$ of cases demonstrate a chromosomal rearrangement involving the $8 q 11-13$ region. ${ }^{11}$

The differential diagnosis includes rhabdomyosarcoma, hibernoma, and lipoma. Rhabdomyosarcoma is the most common paratesticular mass, and also classically presents as a painless, growing scrotal mass, frequently presenting in the first year of life. ${ }^{6,7}$ Hibernomas are rare tumors derived from brown fat, and usually occur in middle-aged adults. ${ }^{12}$ They rarely arise in the scrotum, but can have a similar appearance to lipoblastoma on imaging, and therefore also warrant consideration. ${ }^{12}$ Lipomas are the most common adipocytic tumor in children, and also share similar imaging finding with lipoblastoma. ${ }^{13}$

US with color Doppler is the preferred initial imaging modality for any intrascrotal mass, although CEUS and sonoelastography have recently demonstrated promise as adjunct modalities. ${ }^{10,14}$ Lipoblastomas usually demonstrate a homogenously hyperechoic, well-circumscribed paratesticular mass with scant blood flow on Doppler. ${ }^{3,9,10}$ However, they may also have focal hypoechoic regions on US, with areas of increased vascularity, owing to their myxoid component. ${ }^{9,10}$ As a general rule, the younger the patient, the greater the myxoid component, and the greater the vascularity. ${ }^{11}$ Likewise, CEUS may demonstrate heterogenous enhancement due to higher vascularity of the myxoid component of the tumor. ${ }^{11,15}$ Sonoelastography of lipoblastoma typically demonstrates an elastic pattern as seen with other adipocytic tumors, in contrast with malignant neoplasms, which virtually always typically appear firmer. ${ }^{10,14}$

Lipomas and hibernomas may have similar sonographic findings, owing to their fatty composition, but with several key differences. Unlike lipoblastomas, lipomas are more consistently homogenously echogenic, avascular, nonenhancing lesions that are well defined, and do not have posterior acoustic enhancement. ${ }^{11,13}$ Bright linear interfaces that separate fat lobules may be present. ${ }^{13}$ While hibernomas have a similarly echogenic appearance on US, they have increased vascularity compared with lipoblastomas or lipomas, related to the increased metabolic demand of brown fat. This is well demonstrated by their avid ${ }^{18} \mathrm{~F}$-FDG uptake on positron emission tomography/computed tomography. ${ }^{11,16}$ By contrast, rhabdomyosarcomas demonstrate large, illdefined, heterogeneously echogenic masses due to 


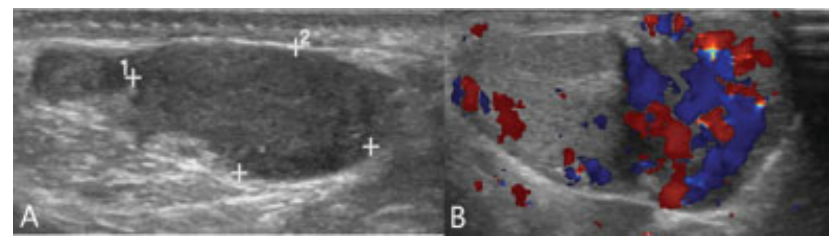

Fig. 3 (A, B) A separate case of an intrascrotal mass in a 3-year-old boy from our institution demonstrates a circumscribed, heterogeneous mass on ultrasound (A) with increased Doppler flow (B) separate from the testicle. This mass was found to be a rhabdomyosarcoma on pathology.

hemorrhage, cystic degeneration, and necrosis. ${ }^{7}$ Hypervascularity relative to the testicle on Doppler ( - Fig. 3 ) and contrast-enhanced US are hallmarks of the malignancy. ${ }^{8,10}$

MRI is the next modality to consider in cases that are inconclusive on US, and is the gold standard for determining the composition and extent of tumor. ${ }^{9,10,17}$ MRI usually demonstrates heterogeneous intensity on T2- and T1weighted imaging (T2WI and T1WI), owing to its variable portion of mature fat. ${ }^{9,15,17}$ There is marked heterogeneous enhancement of the nonfat portion on MRI, similar to CEUS. ${ }^{15}$ Hibernomas have similar MR appearance of increased signal intensity, not quite equal to subcutaneous fat, with increased vascularity that may be well demonstrated on MR angiography. ${ }^{16}$ By comparison, lipomas are more homogenously T1 and T2 intense, and do not enhance. ${ }^{13}$ MRI of rhabdomyosarcoma has characteristic but nonspecific findings, and demonstrates intermediate to hypointense signal on T1WI, and intermediate to hyperintense signal on T2WI, with marked enhancement. ${ }^{7,15,17}$ MRI with fat suppression can be helpful in cases of diagnostic uncertainty between rhabdomyosarcoma and adipocytic tumors, as adipocytic tumors will show an attenuated signal. ${ }^{6}$ If rhabdomyosarcoma is suspected, MRI should be performed to determine the stage of disease before surgery.

Overlapping imaging characteristics of rhabdomyosarcomas and lipoblastomas include their paratesticular location, solid nature, and hypervascularity compared with lipomas. ${ }^{10,11,13}$ Moreover, overlapping patient demographics can make the preoperative diagnosis of lipoblastoma exceedingly difficult. Lipoblastomas have been mistaken for rhabdomyosarcomas and hemangiomas in previous case reports, even when MRI is employed. ${ }^{18}$

The key features that differentiate lipoblastoma from rhabdomyosarcoma include its decreased vascularity on Doppler US, higher elasticity on sonoelastography, reduced enhancement on both CEUS and MRI, and fat suppression MRI sequences. The correct diagnosis is critical, as rhabdomyosarcomas require radical orchiectomy via an inguinal approach, whereas lipoblastomas should be excised without orchiectomy, via a less invasive scrotal approach if the diagnosis is known before surgery. ${ }^{3}$ In ambiguous cases, frozen section analysis should be done intraoperatively to diagnose the mass, which can potentially prevent orchiectomy if lipoblastoma is confirmed. ${ }^{19}$ In our case, addition of MRI, CEUS, sonoelastography, or intraoperative frozen section may have assisted in ruling out rhabdomyosarcoma, and prevented orchiectomy.

\section{Conclusion}

Lipoblastoma should be considered in the differential diagnosis of a growing intrascrotal mass, especially in patients younger than 3 years. Although it is a benign entity, it can be difficult to differentiate from malignant paratesticular rhabdomyosarcoma due to overlapping imaging characteristics and patient demographics. Total excision with preservation of vital organs is the gold standard treatment. Increased awareness of this entity and use of additional imaging modalities such as CEUS, sonoelastography, and MRI, as well as intraoperative frozen section analysis may prevent unnecessary orchiectomy. The prognosis is excellent even with large tumor size and local invasion.

\section{Declaration of Patient Consent}

The authors certify that they have obtained all appropriate patient consent forms. In the form, the patient(s) has/have given his/her/their consent for his/her/their images and other clinical information to be reported in the journal. The patients understand that their names and initials will not be published and due efforts will be made to conceal their identity, but anonymity cannot be guaranteed.

\section{Financial Support and Sponsorship}

Nil.

\section{Conflict of Interest}

None declared.

\section{Acknowledgments}

The views expressed in this manuscript are those of the authors, and do not reflect the official policy or position of the Department of the Army, Department of Defense, or the U.S. government. The authors have no financial, personal, or other vested interests in the information contained within this document.

\section{References}

1 Abdul-Ghafar J, Ahmad Z, Tariq MU, Kayani N, Uddin N. Lipoblastoma: a clinicopathologic review of 23 cases from a major tertiary care center plus detailed review of literature. BMC Res Notes 2018;11(01):42

2 Chung EB, Enzinger FM. Benign lipoblastomatosis. An analysis of 35 cases. Cancer 1973;32(02):482-492

3 Yada K, Ishibashi H, Mori H, Shimada M. Intrascrotal lipoblastoma: report of a case and the review of literature. Surg Case Rep 2016;2(01):34

4 Pereira-Lourenço MJ, Vieira-Brito D, Peralta JP, Castelo-Branco N. Intrascrotal lipoblastoma in adulthood. BMJ Case Rep 2019;12 (12):e231320

5 Hanine D, Aboulam Z, Bouljrouf J, Kisra M. Para testicular lipoblastoma in children (about an exceptional case). Int J Med Res 2018;3:101-103 
6 Akbar SA, Sayyed TA, Jafri SZH, Hasteh F, Neill JSA. Multimodality imaging of paratesticular neoplasms and their rare mimics. Radiographics 2003;23(06):1461-1476

7 Ahmed HU, Arya M, Muneer A, Mushtaq I, Sebire NJ. Testicular and paratesticular tumours in the prepubertal population. Lancet Oncol 2010;11(05):476-483

8 Annam A, Munden MM, Mehollin-Ray AR, Schady D, Browne LP. Extratesticular masses in children: taking ultrasound beyond paratesticular rhabdomyosarcoma. Pediatr Radiol 2015;45(09): 1382-1391

9 Moholkar S, Sebire NJ, Roebuck DJ. Radiological-pathological correlation in lipoblastoma and lipoblastomatosis. Pediatr Radiol 2006;36(08):851-856

10 Sangüesa C, Veiga D, Llavador M, Serrano A. Testicular tumours in children: an approach to diagnosis and management with pathologic correlation. Insights Imaging 2020;11(01):74

11 Burt AM, Huang BK. Imaging review of lipomatous musculoskeletal lesions. SICOT J 2017;3:34

12 Hayashida M, Yano A, Nagamoto S, Sakaguchi K, Okaneya T, Urakami S. Intrascrotal hibernoma mimicking liposarcoma: a case study. Urol Case Rep 2020;32:101206
13 Secil M, Bertolotto M, Rocher L, et al; European Society of Urogenital Radiology Scrotal Imaging Subcommittee. Imaging features of paratesticular masses. J Ultrasound Med 2017;36 (07):1487-1509

14 Aigner F, De Zordo T, Pallwein-Prettner L, et al. Real-time sonoelastography for the evaluation of testicular lesions. Radiology 2012;263(02):584-589

15 Gupta P, Potti TA, Wuertzer SD, Lenchik L, Pacholke DA. Spectrum of fat-containing soft-tissue masses at MR imaging: the common, the uncommon, the characteristic, and the sometimes confusing. Radiographics 2016;36(03):753-766

16 Tomihama RT, Lindskog DM, Ahrens W, Haims AH. Hibernoma: a case report demonstrating usefulness of MR angiography in characterizing the tumor. Skeletal Radiol 2007;36(06):541-545

17 Jalles F, Pinto PL, Martins AP, Goncalves M. Lipoblastoma of the abdominal wall. J Pediatr Surg Case Rep 2019;50:101203

18 Steckman D, Zide B, Greco MA, Rivera R, Blei F. Lipoblastoma of infancy mimicking hemangioma of infancy. Arch Facial Plast Surg 2005;7(05):326-330

19 Dy JS, Fuchs A, Palmer LS. Benign intrascrotal lipoblastoma in a child. Urology 2007;70(02):372.e1-372.e2 